\title{
Greater occipital nerve blockade using ultrasound guidance for the headache of spontaneous intracranial hypotension - A case report -
}

Received July 22, 2021

Revised August 17, 2021

Accepted August 25, 2021

\section{Ji Hee Hong, Ho Woo Lee, and Yong Ho Lee}

Department of Anesthesiology and Pain Medicine, Keimyung University Dong San Hospital, Daegu, Korea

\section{Corresponding author \\ Ji Hee Hong, M.D., Ph.D. \\ Department of Anesthesiology and Pain Medicine, Keimyung University Dong San Hospital, 1035 Dalgubeol- daero, Dalseo-gu, Daegu 42601, Korea}

Tel: 82-53-258-7767

Fax: 82-53-258-6288

E-mail: swon13@daum.net
Background: Spontaneous intracranial hypotension occurs due to cerebrospinal fluid leakage from the spinal column, and orthostatic headache is the most common clinical presentation. Recent studies showed that bilateral greater occipital nerve blockade demonstrated clinical efficacy in relieving post-dural puncture headache after caesarean section.

Case: A 40-year-old male who presented severe orthostatic headache was consulted to our pain clinic from neurology department. He initially felt a dull nature pain over the whole occipital area which then spread over the frontal and parietal areas. His headache was combined with nausea and vomiting. An epidural blood patch was delayed until final cisternography, and bilateral greater occipital nerve blockade using ultrasound guidance was performed instead. After the blockade, the previously existing headache around the occipital and parietal areas disappeared completely, but mild headache persisted around the frontal area.

Conclusions: Greater occipital nerve blockade could be a good therapeutic alternative to improve headache resulting from spontaneous intracranial hypotension.

Keywords: Greater occipital nerve; Orthostatic headache; Spontaneous intracranial hypotension; Ultrasound.
Spontaneous intracranial hypotension (SIH) occurs due to cerebrospinal fluid (CSF) leakage from the spinal column. Underlying dural sac weakness associated with meningeal diverticula, dural weakness caused by minor trauma, ventral dural tears of intervertebral disc material, and CSF-venous fistulas are common causes of SIH. However, in some cases, the exact cause of SIH is completely unknown [1,2]. Orthostatic headache is the most common and important manifestation of SIH patients. Various clinical presentations such as nausea, vomiting, photophobia, hearing impairment and dizziness can be observed with orthostatic headache [1]. Di- agnostic measures for suspected SIH are radioisotope (RI) cisternography, computed tomography myelography, and magnetic resonance imaging (MRI) myelography [1,2].

Conservative treatment for headache due to SIH includes bed rest, theophylline, intravenous fluid infusion, caffeine, and an epidural blood patch (EBP). Among such treatment, an EBP is considered the treatment of choice for those patients whose headache did not improve with initial conservative treatment.

Greater occipital nerve (GON) blockade has been performed widely to relieve symptoms of migraine, tension-type

This is an Open Access article distributed under the terms of the Creative Commons Attribution Non-Commercial License (http://creativecommons.org/licenses/by-nc/4.0) which permits unrestricted non-commercial use, distribution, and reproduction in any medium, provided the original work is properly cited.

Copyright (C) the Korean Society of Anesthesiologists, 2022 
headache, and cluster headache [3]. Recent studies showed that bilateral GON blockade demonstrated clinical efficacy in relieving post-dural puncture headache after caesarean sections [4].

We encountered a patient with severe orthostatic headache who was diagnosed as SIH after admission to the neurology department. Herein, we report this case as GON blockade using ultrasound guidance was performed successfully, and it demonstrated a dramatic improvement of the headache. Before the preparation of this case report, written informed consent for publication was obtained from the patient.

\section{CASE REPORT}

A 40-year-old male with a height of $173 \mathrm{~cm}$ and a weight of $68 \mathrm{~kg}$, who presented severe orthostatic headache, was consulted to our pain clinic from neurology department. The headache started three weeks prior to the admission with a characteristic orthostatic nature. The patient had no history of headache prior to this ongoing orthostatic headache. He initially felt a pain over the whole occipital area which then spread over the frontal and parietal areas. He described his headache as dull nature pain accompanied by pressure sensation, and it was associated with nausea and vomiting. He graded his headache as $9 / 10$ on a numerical rating scale (NRS) before admission to the neurology department. After strict bed rest and intravenous hydration therapy, his NRS decreased to $7 / 10$. The headache persisted for whole day during his admission. Physical examination did not reveal any specific neurologic abnormality or symptoms. He did not have any special history of trauma or accidental dural puncture prior to the onset of this headache. He showed slightly decreased kidney function due to IgA nephropathy which was diagnosed three years ago. He could not maintain his daily activity due to headache. Contrast enhanced MRI revealed bilateral symmetric curvilinear dural enhancement along both cerebral high convexities. Both internal carotid artery angiography and vertebral artery angiography were performed, but they did not demonstrate any suspicious lesion of cerebral venous thrombosis. To identify the leakage level of CSF, cisternography was planned. However, cisternography was only possible at least three weeks later due to an overloaded schedule.

Before the planned cisternography, this patient was consulted to our pain clinic for an EBP. Since we thought that targeted EBP after confirmation of leakage level of CSF is more appropriate for headache improvement, an EBP was delayed until the cisternography was performed. Instead of an EBP, we suggested a bilateral GON blockade using ultrasound guidance for symptom improvement.

The GON blockade was performed by a pain physician who had more than five years of experience in ultrasound guided procedures. The patient was asked to lie with his face down and his head was flexed slightly. Afterward, the spinous process of C2 was palpated and marked. Sterile draping was done using a povidone swab. A linear probe with a frequency of 4-15 MHz was used (Logiq S8, GE Healthcare, USA). After identifying the spinous process of $\mathrm{C} 2$, we placed the probe in a parallel direction to the laminae of $\mathrm{C} 2$ and then rotated it along the long axis of the obliquus capitis inferior (OCI) (Fig 1A). Since the GON always passes by the OCI, the OCI muscle was first identified [5]. After this identification, our final target site was the intermuscular fatty layer between the OCI and semispinalis capitis (SSC) muscle (Fig. 1B). Local infiltration using $1 \%$ lidocaine was done before needle insertion. A $23 \mathrm{G}$ needle was inserted slowly toward the intermuscular fatty layer between the OCI and SSC using an in-plane method. When the needle tip was confirmed to have reached the final target site, $0.1 \%$ ropivacaine $3 \mathrm{ml}$ was injected. We could check the accumulation of the local anesthetics at the intermuscular fatty layer (Fig. 1C). Both sides of the GON blockade were performed using the same local anesthetics and technique.

After completing the bilateral GON blockade, the patient was lay down in bed for $20 \mathrm{~min}$ and was monitored for the appearance of any possible side effect. He was asked to sit up for a while in order to confirm the improvement of the orthostatic headache. He reported that the previous headache which existed around the occipital and parietal areas had disappeared completely, but that mild headache persisted at the frontal area. On the next day after the GON blockade, the significant improvement of headache was still maintained and NRS of the headache was approximately 1-2/10. Two days after GON blockade, he was discharged with mild headache at the frontal area. He was fully educated about the importance of absolute bed rest at home to prevent the aggravation of the intracranial hypotension and headache.

Three weeks after his discharge, he was readmitted for the cisternography. The test demonstrated multiple CSF leakages at the upper thoracic spine, and lumbar puncture opening pressure was as low as $5 \mathrm{cmH}_{2} \mathrm{O}$. Although the headache was mild with NRS of 1-2/10, an EBP was performed to pre- 

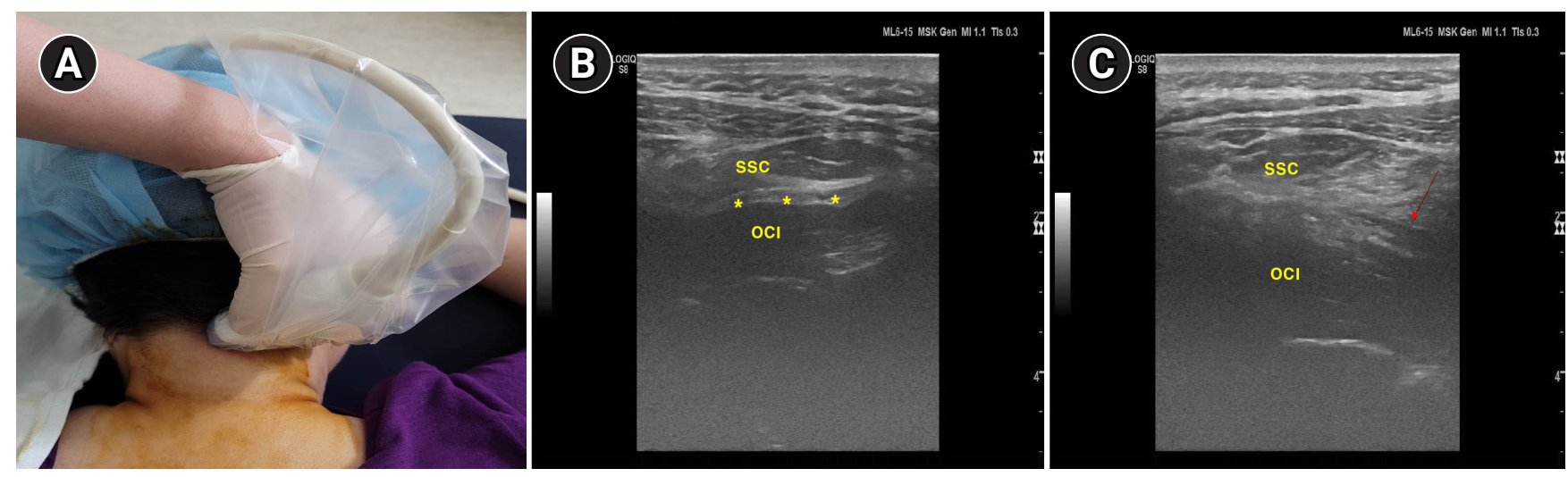

Fig. 1. Photography showing the proper position of the linear probe for greater occipital nerve blockade (A). Ultrasound image showing the obliquus capitis inferior $(\mathrm{OCl})$, and the semispinalis capitis (SSC) muscle. Asterisk (*) indicates intermuscular fatty layer between the OCl and the SSC muscle, which is the final target site (B). Red arrow indicates an accumulation of local anesthetics in the intermuscular fatty layer between the $\mathrm{OCl}$ and the SSC muscle (C).

vent any unwanted complication due to low intracranial pressure. The EBP was performed using the C-arm guided technique. For epidural entry at the specific spinal level, the midline approach via loss-of-air resistance was used. Autologous blood of $10 \mathrm{ml}$ was injected using a 22-G Tuohy epidural needle. The blood was injected very slowly to minimize any discomfort during the injection. Before the blood injection, $3 \mathrm{ml}$ of contrast medium was injected to confirm the epidural space.

One month after the GON blockade and EBP were performed, the orthostatic headache had almost completely disappeared but mild tinnitus remained.

\section{DISCUSSION}

This case of orthostatic headache due to SIH demonstrated a dramatic improvement after bilateral GON blockade using ultrasound guidance. Peripheral nerve blockade including GON has been performed widely to relieve cervicogenic headache, cluster headache, tension-type headache, occipital neuralgia and migraine [3]. The clinical features and mechanism of the headache which occurs due to SIH are distinct compared to common primary headache. Headache of SIH is a clinical result of CSF leakage, leading to a decreased CSF pressure, which eventually triggers a movement of intracranial structures and traction of pain-sensitive structures in the upright position [1,2]. Although such a distinctive nature of the headache is present in SIH, GON blockade resulted in good therapeutic efficacy. There is only one case report which demonstrated an efficacy of GON blockade for the headache due to SIH. GON blockade result- ed in short-term benefit lasting four month, and subsequent pulsed radiofrequency of GON showed significant pain relief lasting 10 months [6].

GON originates from the medial branch of the $\mathrm{C} 2$ dorsal ramus with variable contributions from the $\mathrm{C} 3$ dorsal ramus, and ascends superior to the posterior scalp. GON is always situated between the OCI and SSC muscle and then pierces to the SSC muscle [7]. The trigeminal and upper cervical nerve provide sensory innervation to the face and scalp. The trigeminal nucleus caudalis is located near the upper cervical spinal neuron. The convergence of sensory input from the upper cervical and trigeminal nerves to the trigeminal nucleus caudalis is present. However, in cases of SIH, the activity in the trigeminal and greater occipital nerves can be increased due to a dural stretch caused by a low intracranial pressure [6]. GON blockade can result in the inhibition of sensory input to the trigeminal nucleus caudalis from upper cervical nerves [8]. A "winding down" of the central sensitization caused by the reduction in afferent input to the trigeminal nucleus caudalis can provide a neuromodulatory effect of the headache $[6,8]$.

Many pain physicians still perform a GON blockade using the conventional approach which depends on the palpation of external anatomic landmarks at the level of the superior nuchal line. However, we should keep in mind that the course of GON presents diverse variations at the level of the superior nuchal line $[7,9]$. We used a proximal approach at the $\mathrm{C} 2$ level suggested by Greher et al. [7] targeting GON superficial to the OCI muscle. Their study revealed that the proximal-level GON blockade showed a higher success rate and was more accurate than the conventional blockade at 
the level of the superior nuchal line [7]. Moreover, proximal-level GON blockade provided more sustained analgesic benefit compared with the distal-level GON blockade [10]. Considering a constant relationship between the GON and OCI muscle [7,8], GON blockade at the C2 level provides many advantages compared to the conventional method at the level of the superior nuchal line.

In this case, we delayed the EBP to perform leakage level targeting EBP afterward. In a retrospective study of EBP, site-directed EBP correlated well with a greater likelihood of first EBP efficacy [11]. SIH and post-dural puncture headache have similar pathophysiologic mechanism and clinical manifestation. However, computed tomography-myelography or radionuclide cisternography often revealed multiple levels of CSF leakage throughout the spine in SIH, and the efficacy of EBP was lower in SIH than in post-dural puncture headache $[1,2,11]$.

An EBP has been the treatment of choice for intractable orthostatic headache due to SIH or post-dural puncture headache. However, there is no consensus about the actual blood volume needed for an EBP. A previous study which was performed in SIH patients demonstrated that an epidural blood volume greater than $22.5 \mathrm{ml}$ resulted in a higher success rate compared to a volume less than $22.5 \mathrm{ml}(67.9 \%$ vs. $47.0 \%$ [ [12]. However, if a higher volume of epidural blood is used, we should consider the possibility of rebound intracranial hypertension. The main clinical manifestation of rebound intracranial hypertension is headache, nausea, vomiting, and blurred vision [13], which makes it difficult to distinguish from the clinical manifestation of SIH. Recently reported fatal complications such as acute vision loss [14] and arachnoiditis [15] secondary to an EBP, raise the necessity for the further alternative treatment options.

If an EBP shows minimal efficacy in headache improvement or an unwanted complication is expected, combining a bilateral GON blockade with an EBP can be a good alternative. However, further studies with a larger patient group are required to confirm the clinical efficacy.

\section{FUNDING}

None.

\section{CONFLICTS OF INTEREST}

No potential conflict of interest relevant to this article was reported.

\section{DATA AVAILABILITY STATEMENT}

All data generated or analyzed during this study are included in this published article.

\section{AUTHOR CONTRIBUTIONS}

Writing - original draft: Ji Hee Hong. Writing - review \& editing: Ji Hee Hong. Investigation: Ji Hee Hong. Software: Yong Ho Lee, Ho Woo Lee. Supervision: Ji Hee Hong.

\section{ORCID}

Ji Hee Hong, https://orcid.org/0000-0002-6679-3088

Ho Woo Lee, https://orcid.org/0000-0002-6533-5421

Yong Ho Lee, https://orcid.org/0000-0003-2545-7819

\section{REFERENCES}

1. Schievink WI. Spontaneous spinal cerebrospinal fluid leaks and intracranial hypotension. JAMA 2006; 295: 2286-96.

2. Upadhyaya P, Ailani J. A review of spontaneous intracranial hypotension. Curr Neurol Neurosci Rep 2019; 19: 22.

3. Bovim G, Sand T. Cervicogenic headache, migraine without aura and tension-type headache. Diagnostic blockade of greater occipital and supra-orbital nerves. Pain 1992; 51: 43-8.

4. Uyar Türkyilmaz E, Camgöz Eryilmaz N, Aydin Güzey N, Moraloğlu Ö. Bilateral greater occipital nerve block for treatment of post-dural puncture headache after caesarean operations. Braz J Anesthesiol 2016; 66: 445-50.

5. Pingree MJ, Sole JS, O’ Brien TG, Eldrige JS, Moeschler SM. Clinical efficacy of an ultrasound-guided greater occipital nerve block at the level of C2. Reg Anesth Pain Med 2017; 42: 99-104.

6. Niraj G, Critchley P, Kodivalasa M, Dorgham M. Greater occipital nerve treatment in the management of spontaneous intracranial hypotension headache: a case report. Headache 2017; 57: 952-5.

7. Greher M, Moriggl B, Curatolo M, Kirchmair L, Eichenberger U. Sonographic visualization and ultrasound-guided blockade of the greater occipital nerve: a comparison of two selective techniques confirmed by anatomical dissection. Br J Anaesth 2010; 104: 637-42.

8. Ryu JH, Shim JH, Yeom JH, Shin WJ, Cho SY, Jeon WJ. Ultrasound-guided greater occipital nerve block with botulinum toxin for patients with chronic headache in the occipital area: a randomized controlled trial. Korean J Anesthesiol 2019; 72: 
479-85.

9. Natsis K, Baraliakos X, Appell HJ, Tsikaras P, Gigis I, Koebke J. The course of the greater occipital nerve in the suboccipital region: a proposal for setting landmarks for local anesthesia in patients with occipital neuralgia. Clin Anat 2006; 19: 332-6.

10. Flamer D, Alakkad H, Soneji N, Tumber P, Peng P, Kara J, et al. Comparison of two ultrasound-guided techniques for greater occipital nerve injections in chronic migraine: a double-blind, randomized, controlled trial. Reg Anesth Pain Med 2019; 44: 595-603.

11. So Y, Park JM, Lee PM, Kim CL, Lee C, Kim JH. Epidural blood patch for the treatment of spontaneous and iatrogenic orthostatic headache. Pain Physician 2016; 19: E1115-22.

12. Wu JW, Hseu SS, Fuh JL, Lirng JF, Wang YF, Chen WT, et al. Fac- tors predicting response to the first epidural blood patch in spontaneous intracranial hypotension. Brain 2017; 140: 34452.

13. Kranz PG, Amrhein TJ, Gray L. Rebound intracranial hypertension: a complication of epidural blood patching for intracranial hypotension. AJNR Am J Neuroradiol 2014; 35: 1237-40.

14. Pagani-Estévez GL, Chen JJ, Watson JC, Leavitt JA. Acute vision loss secondary to epidural blood patch: Terson syndrome. Reg Anesth Pain Med 2016; 41: 164-8.

15. Villani LA, Digre KB, Cortez MM, Bokat C, Rassner UA, Ozudogru SN. Arachnoiditis, a complication of epidural blood patch for the treatment of low-pressure headache: a case report and systematic review. Headache 2021; 61: 244-52. 